\title{
Revisiting MERS-CoV Outbreak Chest Radiographic Initial Findings, Temporal Progression and Correlation to Outcomes: Insights for the COVID-19 Pandemic
}

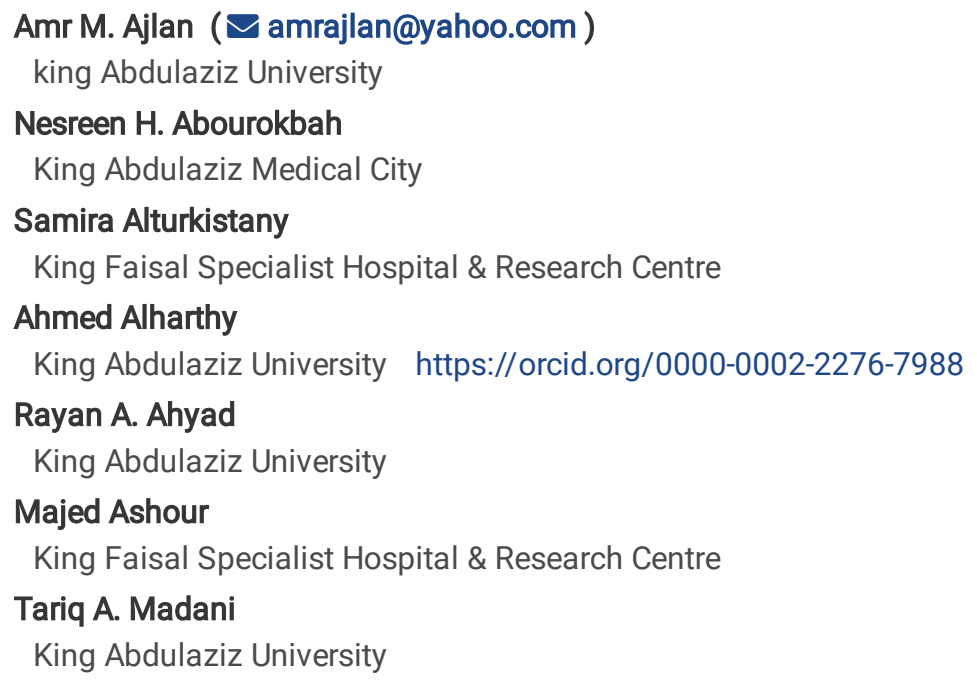




\section{Abstract}

\section{Objective:}

Accounts of initial and follow-up chest X-rays (CXRs) of the Middle East respiratory coronavirus (MERS-CoV) patients, and correlation with outcomes, are sparse. We retrospectively evaluated MERS-CoV CXRs initial findings, temporal progression and outcomes correlation. Such insights may be valuable during the current coronavirus disease 2019 (COVID-19) pandemic, given the clinical and imaging similarities described in both entities.

\section{Methods:}

Fifty-three real-time reverse-transcriptase-polymerase chain reaction (rRT-PCR)-confirmed MERS-CoV patients with CXRs were retrospectively identified from November 2013 to October 2014. Initial and follow-up CXR imaging findings and distribution were evaluated over 75 days. Findings were correlated with outcomes.

\section{Results:}

Twenty-two of 53 (42\%) initial CXRs were normal. In 31 (68\%) abnormal initial CXRs, 15 (48\%) showed bilateral non-diffuse involvement, $16(52 \%)$ had ground-glass opacities (GGO), and 13 (42\%) had peripheral distribution. On follow-up CXRs, mixed airspace opacities prevailed, seen in 16 (73\%) of 22 patients 21-30 days after the initial CXRs. Bilateral non-diffuse involvement was the commonest finding throughout follow-up, affecting 16 (59\%) of 27 patients 11-20 days after the initial CXRs. Bilateral diffuse involvement was seen in 5 (63\%) of 8 patients 31-40 days after the initial CXRs. A bilateral diffuse CXR pattern had an odds ratio for mortality of $13(95 \% \mathrm{Cl}=2-78)$ on worst and $18(95 \% \mathrm{Cl}=3-119)$ on final CXRs (P-value $<0.05)$.

\section{Conclusion:}

Initially, normal CXRs are common in MERS-CoV patients. Peripherally located ground-glass and mixed opacities are common on initial and follow-up imaging. The risk of mortality is higher when bilateral diffuse radiographic abnormalities are detected.

\section{Key Points}

- On initial chest radiographs, imaging was normal in $42 \%$ of patients, while ground-glass and mixed airspace opacities where seen in $52 \%$ and $29 \%$ of cases, respectively.

- Bilateral non-diffuse involvement was the most frequent pattern on chest radiographic follow-up from baseline imaging, seen in $54 \%$ and $59 \%$ patients at $6-10$ days and $11-20$ days, respectively.

- A diffuse bilateral involvement on the worst and final imaged chest radiographs is independently associated with 13-and 18-folds increased mortality risk, respectively.

\section{Introduction}

The Middle East respiratory coronavirus (MERS-CoV) has emerged in Saudi Arabia in 2012 and spread since to 27 countries ${ }^{1,2}$. Up to the end of 2019, the confirmed number of MERS-CoV cases was 2499, with 858 deaths ${ }^{1}$. MERS-CoV, with camels acting as intermediate hosts and a primary source of infection, is mainly spread in the community and healthcare facilities via human-to-human transmission, causing major outbreaks ${ }^{1,3,4}$. Although the last outbreak occurred in Korea in $2015^{3}$, the virus still causes predominantly seasonal clusters in Saudi Arabia, the Middle East and other countries ${ }^{5}$.

Given the current coronavirus disease 2019 (COVID-19) pandemic caused by severe acute respiratory syndrome (SARS) coronavirus - 2 (SARS-CoV-2), we revisited the Jeddah, Saudi Arabia 2013-2014 MERS-CoV outbreak for several reasons. Both coronaviruses currently coexist in the Middle East and around the globe in symptomatic and asymptomatic individuals ${ }^{6-9}$. Symptomatic patients may present with respiratory symptoms, such as cough, fever and dyspnea, and non-respiratory complaints ${ }^{1,6,8}$. The overlap in clinical features and risk of death from either virus heightens the significance of such coexistence ${ }^{1,8,9}$. Despite the public fear of COVID-19, which has a casefatality rate of about $1 \%$, MERS-CoV has a higher case-fatality rate of around $34 \%{ }^{1,9}$. Furthermore, the reported imaging magnifications of both viruses are remarkably similar ${ }^{7-9}$. The utility of chest X-rays (CXRs) in investigating MERS-CoV and COVID-19 is practically 
convenient, yet the number of dedicated studies addressing the CXR findings of both coronaviruses is limited, especially when compared to that of their CT features $6,10-17$.

We retrospectively studied CXR cases from the Jeddah city initial MERS-CoV outbreak to identify the radiographic appearance on the initial CXRs and temporal progression over follow-up studies. We also studied imaging predictors of final patients' outcomes.

\section{Materials And Methods Subjects:}

The local research ethics committees of three participating Jeddah-located hospitals approved this retrospective study, and the consent form was waived. The electronic archives were searched from November 2013 till October 2014 for all cases with a confirmed diagnosis of MERS. Case confirmation was based on at least one positive real-time reverse-transcriptase-polymerase chain reaction (rRT-PCR) respiratory sample, by targeting MERS-CoV RNA upstream region of the E gene and the open reading frame ORF1a and ORF1b regions. Five patients with no chest radiographic imaging, and three patients younger than 18-years of age were excluded. Seven patients included in this study were previously reported, but for CT imaging features and not for CXR findings ${ }^{18}$. The clinical, laboratory, and imaging findings and outcome were analyzed, and initial false negative rRT-PCR results were recorded. Diabetes mellitus, hypertension, chronic lung diseases, cardiac diseases, chronic renal failure, neurological deficits, pregnancy or immunocompromised status were recorded as comorbidities. For outcome data analysis, patients were divided into those deceased versus those who survived; the latter include those transferred to other institutions with no further follow-up data.

\section{Image Analysis:}

Standard-technique chest radiographs were obtained in upright posteroanterior or portable anteroposterior projections using the following systems: Carestream DirectView system (Kodak), OmniDiagnost (Philips), Ysio (Siemens), Mobvision DR unit (Almana Medical Imaging), GE Definium AMX 700 (GE) and Carestream DRX-Revolution. Three fellowship-trained thoracic radiologists, each with 11-12 years of practical experience, reviewed the frontal chest radiographs on Sectra IDS7 (Sectra Medical) and Agfa IMPAX (Agfa Healthcare) picture archiving and communication system workstations.

Applied terminology was in line with the Fleischer Society terms ${ }^{19}$. Airspace opacities were classified as ground-glass opacities (GGO) if underlying lung markings were not obscured, as consolidation if underlying lung markings were obscured, and as mixed opacities, if both patterns coexisted. Interstitial opacities were divided into reticular if linear, nodular if rounded and reticulonodular if both patterns coexisted. The presence and laterality of pneumothoraces and pleural effusions, as well as the presence of cavities (i.e. rounded or oval air- or fluid level-containing lucencies), were sought.

Lung involvement by airspace opacities was categorized as being unilateral or bilateral. The abnormality was assigned craniocaudal or transverse distributions, whenever possible, whether unilateral or bilateral, focal or diffuse. The predominant craniocaudal distribution was categorized as being in the upper lung (i.e. above the hilum), in the lower lung (i.e. below the hilum), or nonspecific (i.e. with equal upper and lower lung involvement). The predominant transverse distribution was categorized as being central (i.e. perihilar), peripheral (i.e. subpleural), both central and peripheral (i.e. if perihilar and subpleural locations are distinctly seen on the same radiograph) or nonspecific (i.e. scattered or diffuse opacities with no distinctive central or peripheral predominance). The abnormality was considered 'focal' if it was unilateral and predominantly confined to the upper or lower lung; and further assigned the following locations: right upper, right lower, left upper or left lower lung.

All available initial and follow-up CXRs were evaluated for the features mentioned above. The presentation CXR (i.e. within the first day) was designated as the 'initial' examination (period I) and was assessed separately. The follow-up radiographs were assessed according to the following periods: >1-5 follow-up days (period II), 6-10 follow-up days (period III), 11-20 follow-up days (period IV), 21-30 follow-up days (period V), 31-40, 41-50 follow-up days, (period VI) and 51-75 follow-up days (period VII). For each patient in each period, the most predominant and persistent findings and distribution were subjectively recorded collectively for all follow-up CXRs performed during that same period. The final follow-up chest radiograph was categorized as normal in a discharged patient, improved in a discharged patient, abnormal in an expired patient, or abnormal in a patient transferred to a non-participating isolation institution.

An overall imaging pattern was summarized for each patient in each period, from better to worse, as follows: normal (i.e. no abnormalities detected), focal opacity (i.e. unilateral airspace involvement of an upper or lower lung location), bilateral non-diffuse opacities (i.e. bilateral 
airspace involvement of clear upper or lower and central or peripheral predominance), or bilateral diffuse opacities (i.e. homogenous or heterogeneous bilateral airspace involvement with no clear craniocaudal or transverse predominance). Additionally, each patient was assigned a worst imaging pattern of involvement according to the period for which it was first seen.

\section{Statistical Analysis:}

Statistical analyses were performed with the Statistical Package for Social Sciences version 21.0 for Windows (SPSS Inc., Chicago, IL, USA). Data are presented as frequencies and percentages for categorical variables, and as means \pm standard deviations (SDs) for continuous variables. The correlation of initial rRT-PCR result with initial CXR patterns was analyzed using $x 2$ testing, with calculation of proportional agreement. Independent t-test was used to compare means and SDs of age (in years) of survived versus deceased patients. Factors of mortality were analyzed by comparing means \pm SDs of age between survived and deceased patients using independent $t$-test, and by comparing the percentage of survived and deceased patients for other factors using $\chi 2$ or Fisher's exact tests, as appropriate. Multivariate binary regression was performed to determine whether a bilateral diffuse pattern on worst and final CXRs -beside other covariates- was an independent factor of mortality; with results presented as odds ratios (OR) with $95 \%$ confidence intervals (Cl). For all calculations, a P-value of $<0.05$ was considered statistically significant.

\section{Results}

\section{Patients' Results:}

Patients' demographics, clinical characteristics and hospital course are detailed in Table 1, and laboratory results are detailed in Table 2. Fifty-three hospitalized patients constituted the study cohort, $33(62 \%)$ of whom were males and $19(36 \%)$ of whom were medical professionals (Table 1). The age range of the total population was 23 to 76 years (mean and SD, $43.7 \pm 15.4$ years). Twenty-one (40\%) patients had one or more comorbidity. Cough and fever were the most frequent symptoms, each encountered in 39 (74\%) patients. The initial symptoms were of non-respiratory complaint in nine (17\%) patients. Superadded bacterial infection occurred in 20 (38\%) patients. Twenty-six (49\%) patients required respiratory support during any of the studied periods. Twenty-nine (55\%) patients were discharged, 15 (28\%) patients died during admission, and $9(17 \%)$ patients were transferred to non-participating institutions.

\section{Imaging Findings:}

\section{General Findings:}

Over the total follow-up period, a sum of 692 CXRs was performed and analyzed (range of 1-55 per patient; mean and SD, $7 \pm 14$ CXRs). Out of 46 patients with a known duration from symptoms onset to the time of obtaining the initial CXR, 17 (37\%) had normal initial CXRs; obtained with a period of one to 34 days (mean and SD, $5.5 \pm 7.7$ days). According to imaged period, chest radiographic detailed findings are summarized in Table 3, while summarized radiograph patterns, radiographic abnormalities distributions and clinical status by followup period are summarized in Table 4.

\section{Initial CXR Imaging Findings:}

On 53 initial CXRs, 31 (58\%) patients were abnormal and 22 (42\%) were normal (figure 1). In the 31 abnormal initial CXRs, findings were bilateral in 18 (58\%) patients (figure 2), of lower lung distribution in $23(74 \%)$ patients, of peripheral in the distribution in $13(42 \%)$ cases, and combined peripheral and central distribution in $7(23 \%)$ cases. Ground-glass opacities were the most common finding, observed in 16 $(52 \%)$ of 31 patients, followed by mixed airspace opacities in nine of $31(29 \%)$ cases. Focal opacities were found in $13(42 \%)$ of 31 patients (figure 3), preferentially involving the lower lobes (10 cases; $77 \%$ ). Pleural effusion was found in 6 (19\%) of 31 cases, while interstitial (i.e. reticular) opacities were only found in one (2\%) of 31 patients (figure 4 ). Initial CXR findings of the nine patients who presented with non-respiratory symptoms showed a normal image in three (33\%), focal opacities in three (33\%), bilateral non-diffuse opacities in two (22\%), and bilateral diffuse opacities in one (11\%) (results not presented in tables). No cavities were detected.

\section{Evolution of CXR Imaging Findings:}


The subsequent imaging periods were marked by increasing frequency of bilateral involvement, reaching 30 of $39(77 \%)$ patients imaged at period III, seven (18\%) of whom had a bilateral diffuse pattern. The proportionally largest percentage of bilateral diffuse involvements was encountered during period V (seven of 22 patients; $32 \%$ ) and period VI (five of eight patients; $63 \%$ ). A bilateral non-diffuse pattern predominated otherwise in most periods, especially at periods II and V, where it constituted $46-59 \%$ of the imaged patients in each period; respectively. As for the type of follow-up findings, mixed airspace opacities predominated throughout, reaching up to 16 (73\%) of 22 patients imaged at period V. Interstitial opacities became relatively more frequent at periods III and VI, with the highest frequency being that of nine $(41 \%)$ of 22 patients encountered at period V. Five (9\%) of 53 patients developed a pneumothorax at different periods of the studied duration. Pleural effusions were infrequent. No cavities were detected.

\section{Worst CXR Imaging Findings:}

Of the 53 patients, the worst CXR imaging finding was first encountered on the initial CXR in 26 (49\%), period III in nine 9 (17\%), period IV in $11(21 \%)$, period $\mathrm{V}$ in three $(6 \%)$, period VI in three $(6 \%)$, and period VII in one (2\%) patients.

\section{Correlation of Initial rRT-PCR Results with Initial CXR Findings:}

The initial rRT-PCR was positive in 31 (58\%) patients and negative in $21(40 \%)$, while one (2\%) patient was not tested. Out of the initial rRTPCT samples, 17 (40\%) of 43 nasopharyngeal swabs and $3(50 \%)$ of six tracheal aspirates returned negative results. The correlation between initial rRT-PCR results (i.e. positive versus negative) and initial CXR findings (i.e. normal versus abnormal) showed $44 \%$ proportional agreement, and there was no statistically significant association between initial rRT-PCR result and severity pattern on initial CXR (P-value of 0.505). Paradoxically, the percentage of abnormal initial CXRs in the initially negative rRT-PCR results group was higher than that in the positive rRT-PCR results group ( $14 / 21$ or $67 \%$ versus $16 / 31$ or $52 \%$, respectively), but the difference was not statistically significant (P-value of 0.281 ). Results for this section are not presented in tables.

\section{Predictors of Patients' Outcome:}

Mortality was significantly associated with both worst and final CXR patterns. That is, no mortality was observed in case of normal or focal opacity CXR patterns images in both the worst and last CXRs. However, a bilateral diffuse CXR pattern in the worst and last CXRs was associated with $62.5 \%$ and $76.9 \%$ mortality, respectively (P-value of 0.001 ). Additionally, patients who died were significantly older (>44 years) compared to survivors at follow-up ( $55 \pm 16$ versus $39 \pm 13$ patients, respectably), with a P-value of 0.001 . The mortality rate was also significantly higher in cases with compared to cases without superadded infection ( $50.0 \%$ versus $15 \%$, respectively), with a Pvalue of 0.011 . No significance was elucidated for either gender or comorbidity.

On separate multivariate regression models, a bilateral diffuse CXR pattern in both worst $(O R=13, P$-value $=0.006)$ and last $(O R=18$, Pvalue $=0.002)$ CXRs were independently associated with patient death; alongside to an age $>44$ years-old, which was highly predictive of death in both groups. However, superadded infection showed no statistical significance on multivariant regression analysis.

\section{Discussion}

Only a few studies have addressed CXR findings of MERS-CoV ${ }^{10,15,20,21}$. Our study and that of Das et al. ${ }^{10}$ are, to the best of our knowledge, the only publications describing the temporal radiographic findings in a relatively large hospitalized population sample. We addressed CXR imaging findings over various periods. Compared to Das et al. ${ }^{10}$, we evaluated patients over a more extended follow-up period and a larger number of analyzed CXRs. We also categorized the overall CXR imaging into simple patterns, in a way that could be easier for physicians to digest and potentially correlate to outcomes.

We encountered $42 \%$ patients with initially normal CXRs, a percentage comparable to that of the study by Hamimi ${ }^{15}$, but different from the $17 \%$ reported otherwise ${ }^{2,8,10}$. In the context of the current pandemic, COVID-19 has also been reported to show initially normal CXRs in about $15-30 \%{ }^{8,22}$.

Our cohort showed almost equal numbers of focal and bilateral non-diffuse initial CXR patterns. In line with other publications ${ }^{2,8,10}$, the normality of CXRs decreased as patients were followed, with an increasing number of bilateral non-diffuse or diffuse lung involvement. We noticed that the worst CXR progression occurred between a week to a month from the time of obtaining the initial CXR. 
GGO, whether pure or mixed with consolidation, was the most common CXR finding in our study. As the abnormalities progressed over time, superimposed consolidation appeared or progressed. Whether abnormalities were seen on initial or follow-up CXRs, the most common distribution was that of bilateral peripheral location, with or without perihilar involvement. Such findings and distribution are another point of similarity for what is currently experienced with COVID-19 8,12,22,23. The predilection for such peculiar distribution has previously raised the suggestion that novel viruses, from the coronavirus family or others, maybe inducing an acute organizing pneumonia reaction $18,24,25$.

Pleural effusions, pneumothoraces, cavities and bronchial wall thinking were all rarely seen in our study, and are not common in the literature of CXR imaging of MERS-CoV 2,8,10,18. A noteworthy observation is that interstitial opacities were also uncommon in our study and other reports. However, reticulations were observed in some patients where longer-term follow-up imaging was obtained ${ }^{8,18,26}$. Reticulations have been noticed, although rarely, in cases of current COVID-19 infection as well ${ }^{27}$. There is a possibility that long-term sequelae of coronaviruses pneumonia include residual fibrosis ${ }^{8,18}$.

Avoidance of causes of false negative rRT-PCR testing has been addressed in MERS-CoV $(21,25)$ but has not been correlated to the initial CXR appearance. In several cases in our study, rRT-PCR was initially falsely negative when the first CXR was abnormal, and vice versa. By combining initial CXR and rRT-PCT results, the detection rate in our cohort would have increased from $59 \%$ to $87 \%$. Of note, the issue of false negative rRT-PCR results is a current hot topic with COVID-19 pneumonia as well, for which several groups have advocated for early chest CT as a way to ameliorate challenges related to rRT-PCR diagnostic performance $23,28,29$.

Prior MERS-CoV research has shown that the risk of mortality significantly increases in proportion to the extent of lung involvement

$2,8,10,26$. Such publications are in concordance with our observation that a bilateral diffuse pattern of lung abnormalities, as observed on the worst or finally imaged CXR, was independently related to higher death rates. On the other hand, no death occurred in patients in whose worst or finally imaged CXRs were normal or focally abnormal. Further, the risk of MERS-CoV mortality in our group was found to be highest when patients where $>44$ years-old. Herein lies another similarity with recent observations of COVID-19, of which older age and more substantial lung involvement are associated with poorer prognosis $8,17,30$.

The expected variability of sampled CXRs per various periods and the decreasing number of CXRs obtained as time progressed reduced the studied samples per specific periods. Furthermore, the study size is considered relatively small for deriving generalized outcome data conclusions. This point is even more relevant when considering the potentially confounding factor of superadded infection, which may by itself, cloud CXR interpretations. Another limitation is that a proportion of patients that were eventually transferred to other institutes that were not included in the study scope were considered survivors.

To conclude, a substantial number of MERS-CoV cases may have a normal initial CXR. When abnormal, bilateral lower-lung-predominant ground-glass or mixed opacities on CXRs were the most prevalent imaging appearance. Worsening CXRs progression occurred within a week to a month from the initial imaging. Bilateral diffuse lung involvement is an independent risk for higher mortality, worsened by older patients. False negative rRT-PCR results may occur whether CXRs were initially normal or abnormal, and combining the results of both CXR and rRT-PCR provides a better diagnostic yield in patients suspected of having MERS-CoV. Finally, such insights are timely, given the various clinical and imaging similarities of MERS-CoV outbreaks with those of the COVID-19 pandemic.

\section{Abbreviations}

MERS-CoV = Middle East respiratory coronavirus, COVID-19 = coronavirus 2019, CXR = chest X-ray, rRT-PCR = real-time reversetranscriptase-polymerase chain reaction, $\mathrm{GGO}=$ ground-glass opacity, $\mathrm{SD}=$ standard deviation, $\mathrm{Cl}=\mathrm{confidence}$ interval

\section{Declarations}

1. Acknowledgements: The authors would like to thank Dr Mohamed Amine Haireche for his guidance in the statistical section and his input on the final manuscript (written permission has been obtained).

2. Funding: The authors state that this work has not received any funding.

\section{Compliance with Ethical Standards}

3. Guarantor: The scientific guarantor of this publication is the team of authors lead by Dr Amr Ajlan. 
4. Conflict of Interest: The authors of this manuscript declare no relationships with any companies, whose products or services may be related to the subject matter of the article.

5. Statistics and Biometry: Simple and well-known calculation were performed via SPSS. No complex statistical methods were necessary for this paper. One of the authors has significant statistical expertise and calculations were checked and refined by the acknowledged Dr Mohamed Amine Haireche.

6. Informed Consent: Written informed consent was waived by the Institutional Review Boards.

7. Ethical Approval: Institutional Review Boards approval was obtained.

8. Study subjects or cohorts overlap: Seven out of the total 53 studied patient population were previously included in this study, but unlike the current paper, were evaluated for CT imaging features and not for chest radiographic findings (such overlap has been stated on the Martials and Methods section and the prior publication PDF has been submitted).

9. Methodology: Retrospective cross-sectional multicentre study

\section{References}

1. Memish ZA, Perlman S, Kerkhove MDV, Zumla A (2020) Middle East respiratory syndrome. Lancet Lond Engl 395:1063-1077. https://doi.org/10.1016/s0140-6736(19)33221-0

2. Das KM, Lee EY, Langer RD, Larsson SG (2016) Middle East Respiratory Syndrome Coronavirus: What Does a Radiologist Need to Know? Am J Roentgenol 206:1193-1201. https://doi.org/10.2214/ajr.15.15363

3. Oh M-D, Park WB, Park S-W, et al (2018) Middle East respiratory syndrome: what we learned from the 2015 outbreak in the Republic of Korea. Korean J Intern Medicine 33:233-246. https://doi.org/10.3904/kjim.2018.031

4. Azhar El, El-Kafrawy SA, Farraj SA, et al (2014) Evidence for camel-to-human transmission of MERS coronavirus. New Engl J Medicine 370:2499-505. https://doi.org/10.1056/nejmoa1401505

5. Al-Ahmadi K, Alahmadi S, Al-Zahrani A (2019) Spatiotemporal Clustering of Middle East Respiratory Syndrome Coronavirus (MERS-CoV) Incidence in Saudi Arabia, 2012-2019. Int J Environ Res Pu 16:2520. https://doi.org/10.3390/ijerph16142520

6. Vetter P, Vu DL, L'Huillier AG, et al (2020) Clinical features of covid-19. Bmj Clin Res Ed 369:m1470. https://doi.org/10.1136/bmj.m1470

7. Kay F, Abbara S (2020) The Many Faces of COVID-19: Spectrum of Imaging Manifestations. Radiology Cardiothorac Imaging 2:e200037. https://doi.org/10.1148/ryct.2020200037

8. Hosseiny M, Kooraki S, Gholamrezanezhad A, et al (2020) Radiology Perspective of Coronavirus Disease 2019 (COVID-19): Lessons From Severe Acute Respiratory Syndrome and Middle East Respiratory Syndrome. Ajr Am J Roentgenol 214:1-5.

https://doi.org/10.2214/ajr.20.22969

9. Petrosillo N, Viceconte G, Ergonul O, et al (2020) COVID-19, SARS and MERS: are they closely related? Clin Microbiol Infect Official Publ European Soc Clin Microbiol Infect Dis. https://doi.org/10.1016/j.cmi.2020.03.026

10. Das KM, Lee EY, Jawder SEA, et al (2015) Acute Middle East Respiratory Syndrome Coronavirus: Temporal Lung Changes Observed on the Chest Radiographs of 55 Patients. Am J Roentgenol 205:W267-S274. https://doi.org/10.2214/ajr.15.14445

11. Yang R, Li X, Liu H, et al (2020) Chest CT Severity Score: An Imaging Tool for Assessing Severe COVID-19. Radiology Cardiothorac Imaging 2:e200047. https://doi.org/10.1148/ryct.2020200047

12. Yoon SH, Lee KH, Kim JY, et al (2019) Chest Radiographic and CT Findings of the 2019 Novel Coronavirus Disease (COVID-19): Analysis of Nine Patients Treated in Korea. Korean J Radiol 21:494-500. https://doi.org/10.3348/kjr.2020.0132

13. Habib AMG, Ali MAE, Zouaoui BR, et al (2019) Clinical outcomes among hospital patients with Middle East respiratory syndrome coronavirus (MERS-CoV) infection. Bmc Infect Dis 19:870. https://doi.org/10.1186/s12879-019-4555-5 
14. Salehi S, Abedi A, Balakrishnan S, Gholamrezanezhad A (2020) Coronavirus Disease 2019 (COVID-19): A Systematic Review of Imaging Findings in 919 Patients. Ajr Am J Roentgenol 1-7. https://doi.org/10.2214/ajr.20.23034

15. Hamimi A (2016) MERS-CoV: Middle East respiratory syndrome corona virus: Can radiology be of help? Initial single center experience. Egypt J Radiology Nucl Medicine 47:95-106. https://doi.org/10.1016/j.ejrnm.2015.11.004

16. Jacobi A, Chung M, Bernheim A, Eber C (2020) Portable chest X-ray in coronavirus disease-19 (COVID-19): A pictorial review. Clin Imag 64:35-42. https://doi.org/10.1016/j.clinimag.2020.04.001

17. Toussie D, Voutsinas N, Finkelstein M, et al (2020) Clinical and Chest Radiography Features Determine Patient Outcomes In Young and Middle Age Adults with COVID-19. Radiology 201754. https://doi.org/10.1148/radiol.2020201754

18. Ajlan AM, Ahyad RA, Jamjoom LG, et al (2014) Middle East Respiratory Syndrome Coronavirus (MERS-CoV) Infection: Chest CT Findings. Am J Roentgenol 203:782-787. https://doi.org/10.2214/ajr.14.13021

19. Hansell DM, Bankier AA, MacMahon H, et al (2008) Fleischner Society: glossary of terms for thoracic imaging. Radiology 246:697-722. https://doi.org/10.1148/radiol.2462070712

20. Assiri A, Al-Tawfiq JA, Al-Rabeeah AA, et al (2013) Epidemiological, demographic, and clinical characteristics of 47 cases of Middle East respiratory syndrome coronavirus disease from Saudi Arabia: a descriptive study. Lancet Infect Dis 13:752-761.

https://doi.org/10.1016/s1473-3099(13)70204-4

21. Memish ZA, Zumla Al, Al-Hakeem RF, et al (2013) Family cluster of Middle East respiratory syndrome coronavirus infections. New Engl J Medicine 368:2487-94. https://doi.org/10.1056/nejmoa1303729

22. Wong HYF, Lam HYS, Fong AH-T, et al (2019) Frequency and Distribution of Chest Radiographic Findings in COVID-19 Positive Patients. Radiology 201160. https://doi.org/10.1148/radiol.2020201160

23. Inui S, Fujikawa A, Jitsu M, et al (2020) Chest CT Findings in Cases from the Cruise Ship "Diamond Princess" with Coronavirus Disease 2019 (COVID-19). Radiology Cardiothorac Imaging 2:e200110. https://doi.org/10.1148/ryct.2020200110

24. Ajlan AM, Quiney B, Nicolaou S, Müller NL (2009) Swine-origin influenza A (H1N1) viral infection: radiographic and CT findings. Ajr Am J Roentgenol 193:1494-9. https://doi.org/10.2214/ajr.09.3625

25. Wu Y, Xie Y, Wang X (2020) Longitudinal CT Findings in COVID-19 Pneumonia: Case Presenting Organizing Pneumonia Pattern. Radiology Cardiothorac Imaging 2:e200031. https://doi.org/10.1148/ryct.2020200031

26. Das KM, Lee EY, Singh R, et al (2017) Follow-up chest radiographic findings in patients with MERS-CoV after recovery. Indian J Radiology Imaging 27:342-349. https://doi.org/10.4103/ijri.ijri_469_16

27. Liu H, Liu F, Li J, et al (2020) Clinical and CT Imaging Features of the COVID-19 Pneumonia: Focus on Pregnant Women and Children. J Infect 80:e7-e13. https://doi.org/10.1016/j.jinf.2020.03.007

28. Feng H, Liu Y, Lv M, Zhong J (2020) A case report of COVID-19 with false negative RT-PCR test: necessity of chest CT. Jpn J Radiol 38:409-410. https://doi.org/10.1007/s11604-020-00967-9

29. Xie X, Zhong Z, Zhao W, et al (2020) Chest CT for Typical 2019-nCoV Pneumonia: Relationship to Negative RT-PCR Testing. Radiology 200343. https://doi.org/10.1148/radiol.2020200343

30. Shi H, Han X, Jiang N, et al (2020) Radiological findings from 81 patients with COVID-19 pneumonia in Wuhan, China: a descriptive study. Lancet Infect Dis 20:425-434. https://doi.org/10.1016/s1473-3099(20)30086-4

\section{Tables}

Table 1. Patients' demographics, clinical characteristics and hospital course 


\begin{tabular}{|c|c|}
\hline Parameter & Value \\
\hline \multicolumn{2}{|c|}{ Demographics and clinical characteristics } \\
\hline Age (years) a & $43.7( \pm 15.4)$ \\
\hline \multicolumn{2}{|l|}{ Age category (years) } \\
\hline$\leq 44$ & $31(58 \%)$ \\
\hline$>44$ & $22(42 \%)$ \\
\hline \multicolumn{2}{|l|}{ Sex } \\
\hline Male & $33(62 \%)$ \\
\hline Female & $20(38 \%)$ \\
\hline \multicolumn{2}{|l|}{ Contact history } \\
\hline None & $21(40 \%)$ \\
\hline Confirmed & $23(43 \%)$ \\
\hline Unclear in records & $9(17 \%)$ \\
\hline \multicolumn{2}{|l|}{ Smoking history } \\
\hline Yes & $11(20 \%)$ \\
\hline No & $42(80 \%)$ \\
\hline \multicolumn{2}{|c|}{ Significant comorbidities } \\
\hline Present & $21(40 \%)$ \\
\hline None & $32(60 \%)$ \\
\hline \multicolumn{2}{|l|}{ Profession } \\
\hline Medical & $19(36 \%)$ \\
\hline Others & $34(46 \%)$ \\
\hline \multicolumn{2}{|l|}{ Presenting symptoms ${ }^{b}$} \\
\hline Cough & $39(74 \%)$ \\
\hline Fever & $39(74 \%)$ \\
\hline Myalgia & $19(36 \%)$ \\
\hline Abdominal pain & $10(19 \%)$ \\
\hline Diarrhea & $7(13 \%)$ \\
\hline Dyspnea & $28(53 \%)$ \\
\hline Chest pain & $8(15 \%)$ \\
\hline Rhinitis & $4(8 \%)$ \\
\hline Nausea and vomiting & $7(13 \%)$ \\
\hline Headache & $12(23 \%)$ \\
\hline Hemoptysis & $5(9 \%)$ \\
\hline \multicolumn{2}{|c|}{ Type of initial symptoms at presentation } \\
\hline Respiratory & $44(83 \%)$ \\
\hline Non-respiratory & $9(17 \%)$ \\
\hline Hospital course & \\
\hline
\end{tabular}




\begin{tabular}{|l|l|}
\hline Period from symptoms onset to hospital presentation (days) ${ }^{\text {a }}$ & $5.8( \pm 4.6)$ \\
\hline Duration of admission (days) $^{\text {a }}$ & $22.4( \pm 27.9)$ \\
\hline Superadded infection & $33(62 \%)$ \\
\hline Yes & $20(38 \%)$ \\
\hline No & \\
\hline Administered medications ${ }^{\text {b }}$ & $39(74 \%)$ \\
\hline Antiviral & $43(81 \%)$ \\
\hline Steroids & $21(40 \%)$ \\
\hline Antibiotics & $14(26 \%)$ \\
\hline Interferon & \\
\hline Admission location & $29(55 \%)$ \\
\hline ICU & $24(45 \%)$ \\
\hline Others & \\
\hline Respiratory support & $27(51 \%)$ \\
\hline None & $7(13 \%)$ \\
\hline Intubation & $17(32 \%)$ \\
\hline BiPAP & $2(4 \%)$ \\
\hline ECMO & $29(55 \%)$ \\
\hline Clinical Outcome & $9(17 \%)$ \\
\hline Discharged & \\
\hline Transferred to another facility ${ }^{c}$ & \\
\hline Death & \\
\hline & \\
\hline
\end{tabular}

${ }^{a}$ Calculations are means (and standard deviations), whereas the rest of the table parameters are calculated as frequencies (and percentages).

${ }^{b}$ Frequencies (and percentages) are higher than the population sum total (and 100\%) due to parameter overlap in patients.

${ }^{\mathrm{c}}$ Patients eventually transferred to health care centre other than the three hospitals subject to the study.

$I C U=$ intensive care unit. BiPAP $=$ bi-level positive airway pressure. $E C M O=$ extracorporeal membrane oxygenation.

Table 2. Patients' laboratory results at presentation 


\begin{tabular}{|c|c|c|}
\hline Parameter & & Value \\
\hline \multirow[t]{4}{*}{ Initial specimen type ${ }^{a}$} & Nasopharyngeal swab & $43(83 \%)$ \\
\hline & Tracheal aspirate & $6(12 \%)$ \\
\hline & Bronchoalveolar lavage & $2(4 \%)$ \\
\hline & Induced sputum & $1(2 \%)$ \\
\hline \multirow{3}{*}{$\begin{array}{l}\text { White blood cells } \\
\text { (Normal }=4-11 \times 10^{3} \text { cells per } \mathrm{mm}^{3} \text { of blood) }\end{array}$} & Normal & $23(43 \%)$ \\
\hline & Elevated & $10(19 \%)$ \\
\hline & Low & $20(38 \%)$ \\
\hline \multirow{3}{*}{$\begin{array}{l}\text { Lymphocytes }^{\text {a }} \\
\text { (Normal }=1.5-4 \times 10^{3} \text { cells per } \mathrm{mm}^{3} \text { of blood) }\end{array}$} & Normal & $10(19 \%)$ \\
\hline & Elevated & $1(2 \%)$ \\
\hline & Low & $41(79 \%)$ \\
\hline \multirow{3}{*}{$\begin{array}{l}\text { Eosinophils } \\
\text { (Normal }=1-7 \times 110^{3} \text { cells per } \mathrm{mm}^{3} \text { of blood) }\end{array}$} & Normal & $9(17 \%)$ \\
\hline & Elevated & $40(76 \%)$ \\
\hline & Low & $4(8 \%)$ \\
\hline \multirow{2}{*}{$\begin{array}{l}\text { Hemoglobin } \\
\text { (Normal = 13.0-18.0 gm/dL) }\end{array}$} & Normal & $27(51 \%)$ \\
\hline & Low & $26(50 \%)$ \\
\hline \multirow{3}{*}{$\begin{array}{l}\text { Platelets } \\
\text { (Normal }=150-450 \times 10^{3} \text { cells per } \mathrm{mm}^{3} \text { of blood) }\end{array}$} & Normal & $23(43 \%)$ \\
\hline & Elevated & $3(6 \%)$ \\
\hline & Low & $27(51 \%)$ \\
\hline \multirow{3}{*}{$\begin{array}{l}\text { Lactate } \\
\text { dehydrogenase }^{\mathrm{a}} \\
(\text { Normal }=125-243 \mathrm{U} / \mathrm{L})\end{array}$} & Normal & $6(16 \%)$ \\
\hline & Elevated & $31(81 \%)$ \\
\hline & Low & $1(3 \%)$ \\
\hline \multirow{3}{*}{$\begin{array}{l}\text { Alanine aminotransferase }{ }^{a} \\
\text { (Normal }=5-55 \mathrm{U} / \mathrm{L})\end{array}$} & Normal & $27(54 \%)$ \\
\hline & Elevated & $22(44 \%)$ \\
\hline & Low & $1(2 \%)$ \\
\hline \multirow{3}{*}{$\begin{array}{l}\text { Aspartate aminotransferase }{ }^{a} \\
(\text { Normal }=5-34 \mathrm{U} / \mathrm{L})\end{array}$} & Normal & $12(24 \%)$ \\
\hline & Elevated & $37(73 \%)$ \\
\hline & Low & $2(4 \%)$ \\
\hline \multirow{3}{*}{$\begin{array}{l}\text { Creatine kinase }^{\mathrm{a}} \\
(\text { Normal }=30-200 \mathrm{IU} / \mathrm{L})\end{array}$} & Normal & $11(31 \%)$ \\
\hline & Elevated & $21(60 \%)$ \\
\hline & Low & $3(9 \%)$ \\
\hline \multirow{3}{*}{$\begin{array}{l}\text { Creatinine }^{\mathrm{a}} \\
\text { (Normal }=60-115 \mu \mathrm{mol} / \mathrm{L})\end{array}$} & Normal & $29(56 \%)$ \\
\hline & Elevated & $18(35 \%)$ \\
\hline & Low & $5(10 \%)$ \\
\hline
\end{tabular}

Note: Values are represented in frequency and percentage.

${ }^{a}$ Frequencies (and percentages) are less than the population sum total (and 100\%) due to parameter unavailability/uncertainty in the hospital records. 
Table 3. Patients' detailed chest radiograph findings according to imaging periods ${ }^{\text {a }}$

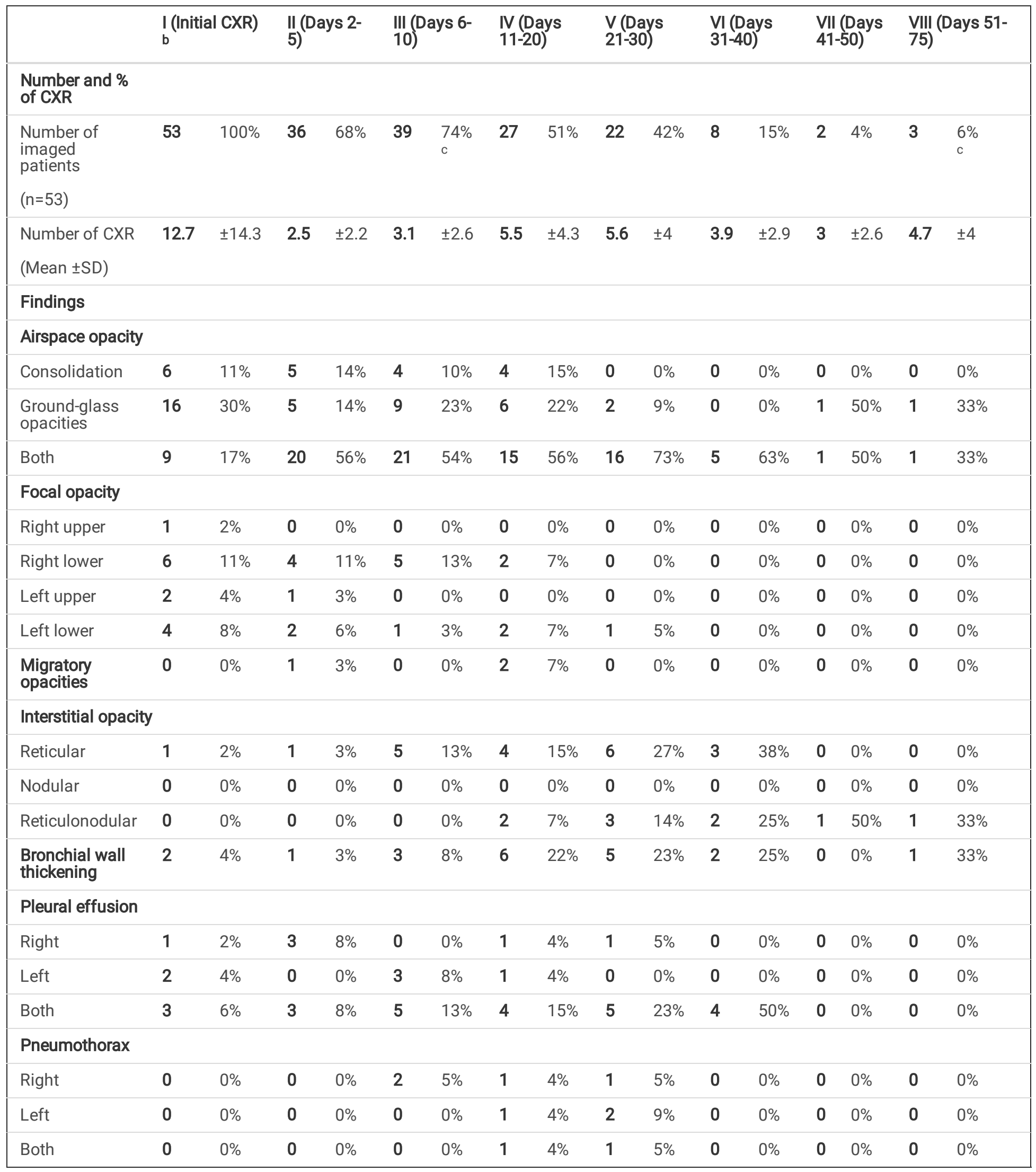

Note: Values are represented in numbers (n) and percentages (\%) unless otherwise indicated.

a Periods are relative to the time of obtaining the initial CXR.

${ }^{\mathrm{b}}$ Period I is within one the first day of obtaining the initial CXR. 
${ }^{\mathrm{c}}$ Periods with increasing instead of decreasing patients' numbers due to fluctuation in the number of imaged patients per period.

CXR $=$ chest $\mathrm{X}$-ray

Table 4. Summarized chest radiograph patterns, abnormalities distribution and clinical status by follow up periods ${ }^{\text {a }}$

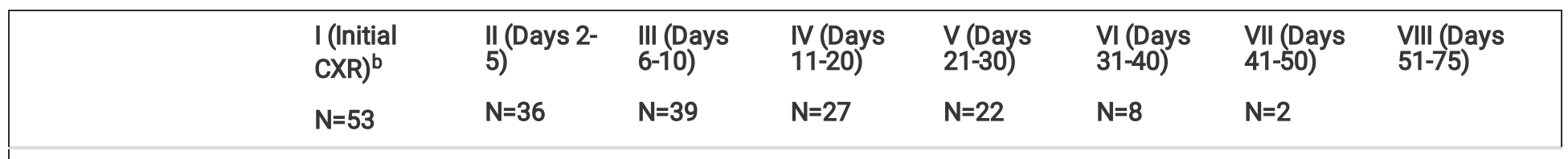

$\mathrm{N}=3$

\section{Summarized CXR pattern}

\begin{tabular}{|c|c|c|c|c|c|c|c|c|c|c|c|c|c|c|c|c|}
\hline Normal & 22 & $42 \%$ & 6 & $17 \%$ & 5 & $13 \%$ & 1 & $4 \%$ & 4 & $18 \%$ & 2 & $25 \%$ & 0 & $0 \%$ & 1 & $33 \%$ \\
\hline Focal opacity & 13 & $25 \%$ & 7 & $19 \%$ & 6 & $15 \%$ & 4 & $15 \%$ & 1 & $5 \%$ & 0 & $0 \%$ & 0 & $0 \%$ & 0 & $0 \%$ \\
\hline $\begin{array}{l}\text { Bilateral non-diffuse } \\
\text { opacities }\end{array}$ & 15 & $28 \%$ & 17 & $47 \%$ & 21 & $54 \%$ & 16 & $59 \%$ & 10 & $46 \%$ & 1 & $13 \%$ & 1 & $50 \%$ & 1 & $33 \%$ \\
\hline $\begin{array}{l}\text { Bilateral diffuse } \\
\text { opacities }\end{array}$ & 3 & $6 \%$ & 6 & $17 \%$ & 7 & $18 \%$ & 6 & $22 \%$ & 7 & $32 \%$ & 5 & $63 \%$ & 1 & $50 \%$ & 1 & $33 \%$ \\
\hline \multicolumn{17}{|l|}{ Distribution } \\
\hline \multicolumn{17}{|l|}{ Axial distribution } \\
\hline Central & 1 & $2 \%$ & 0 & $0 \%$ & 0 & $0 \%$ & 0 & $0 \%$ & 0 & $0 \%$ & 0 & $0 \%$ & 0 & $0 \%$ & 0 & $0 \%$ \\
\hline Peripheral & 13 & $25 \%$ & 5 & $14 \%$ & 7 & $18 \%$ & 3 & $11 \%$ & 2 & $9 \%$ & 0 & $0 \%$ & 1 & $50 \%$ & 1 & $33 \%$ \\
\hline Both & 7 & $13 \%$ & 6 & $17 \%$ & 8 & $21 \%$ & 9 & $33 \%$ & 6 & $27 \%$ & 1 & $13 \%$ & 0 & $0 \%$ & 0 & $0 \%$ \\
\hline Unclassified & 10 & $19 \%$ & 19 & $53 \%$ & 19 & $49 \%$ & 14 & $52 \%$ & 10 & $46 \%$ & 5 & $63 \%$ & 1 & $50 \%$ & 1 & $33 \%$ \\
\hline \multicolumn{17}{|l|}{ Coronal distribution } \\
\hline Upper & 3 & $6 \%$ & 1 & $3 \%$ & 0 & $0 \%$ & 0 & $0 \%$ & 0 & $0 \%$ & 0 & $0 \%$ & 0 & $0 \%$ & 0 & $0 \%$ \\
\hline Lower & 23 & $43 \%$ & 22 & $61 \%$ & 24 & $62 \%$ & 18 & $67 \%$ & 10 & $46 \%$ & 1 & $13 \%$ & 1 & $50 \%$ & 1 & $33 \%$ \\
\hline Unclassified & 5 & $9 \%$ & 7 & $20 \%$ & 10 & $26 \%$ & 8 & $30 \%$ & 8 & $36 \%$ & 5 & $63 \%$ & 1 & $50 \%$ & 1 & $33 \%$ \\
\hline \multicolumn{17}{|l|}{ Side of involvement } \\
\hline Unilateral & 13 & $25 \%$ & 7 & $19 \%$ & 6 & $15 \%$ & 4 & $15 \%$ & 1 & $5 \%$ & 0 & $0 \%$ & 0 & $0 \%$ & 0 & $0 \%$ \\
\hline Bilateral & 18 & $34 \%$ & 23 & $64 \%$ & 28 & $72 \%$ & 22 & $82 \%$ & 17 & $77 \%$ & 6 & $75 \%$ & 2 & $100 \%$ & 2 & $67 \%$ \\
\hline \multicolumn{17}{|l|}{ Clinical status } \\
\hline Hospitalized & 47 & $89 \%$ & 32 & $89 \%$ & 27 & $69 \%$ & 19 & $70 \%$ & 8 & $36 \%$ & 2 & $25 \%$ & 2 & $100 \%$ & 0 & $0 \%$ \\
\hline $\begin{array}{l}\text { Discharged (with } \\
\text { normal CXR) }\end{array}$ & 4 & $8 \%$ & 2 & $6 \%$ & 3 & $8 \%$ & 2 & $7 \%$ & 3 & $14 \%$ & 1 & $13 \%$ & 0 & $0 \%$ & 1 & $33 \%$ \\
\hline $\begin{array}{l}\text { Transferred (with } \\
\text { abnormal CXR) }\end{array}$ & 1 & $2 \%$ & 1 & $3 \%$ & 2 & $5 \%$ & 1 & $4 \%$ & 2 & $9 \%$ & 0 & $0 \%$ & 0 & $0 \%$ & 2 & $67 \%$ \\
\hline Died & 0 & $0 \%$ & 1 & $3 \%$ & 2 & $5 \%$ & 3 & $11 \%$ & 5 & $23 \%$ & 4 & $50 \%$ & 0 & $0 \%$ & 0 & $0 \%$ \\
\hline $\begin{array}{l}\text { Discharged (improved } \\
\text { CXR) }\end{array}$ & 1 & $2 \%$ & 0 & $0 \%$ & 5 & $13 \%$ & 2 & $7 \%$ & 4 & $18 \%$ & 1 & $13 \%$ & 0 & $0 \%$ & 0 & $0 \%$ \\
\hline
\end{tabular}

Note: Values are represented in numbers (n) and percentages (\%) unless otherwise indicated.

a Periods are relative to the time of obtaining the initial CXR. 
${ }^{b}$ Period I is within one the first day of obtaining the initial CXR.

${ }^{\mathrm{c}}$ Periods with increasing instead of decreasing patients' numbers due to fluctuation in the number of imaged patients per period.

$\mathrm{CXR}=$ chest $\mathrm{X}$-ray

\section{Figures}
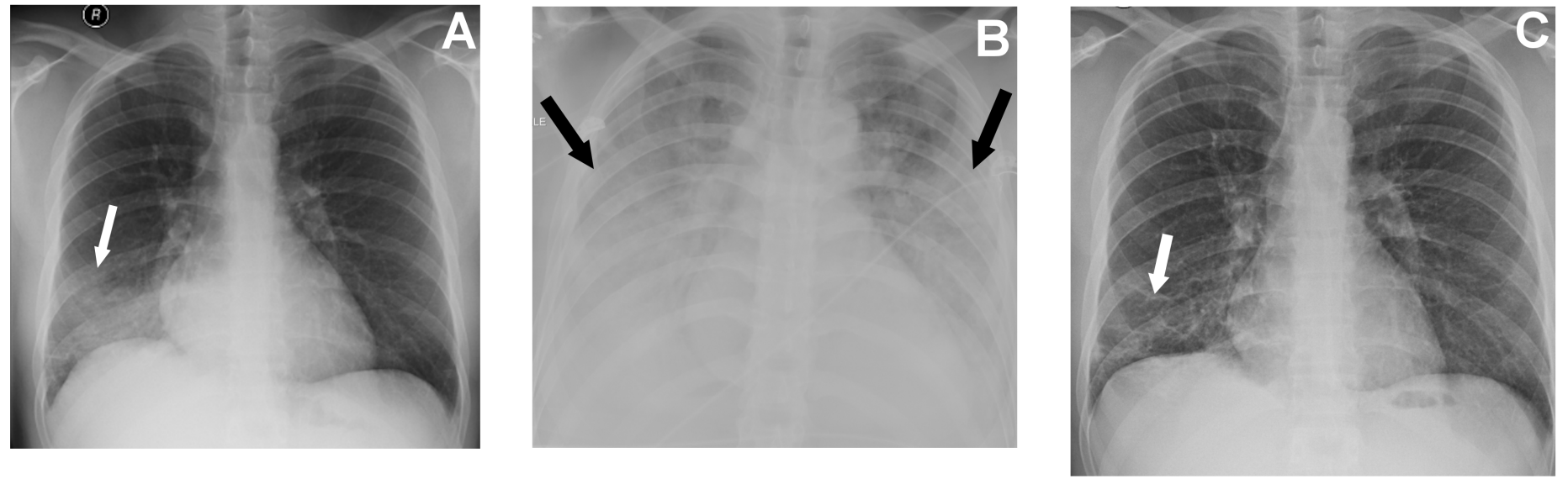

\section{Figure 1}

A 27-year-old medical professional male survivor of MERS-CoV infection, with an initial false-negative PCR result. (A) Initial CXR obtained at ten days from symptoms onset, with focal right lower lung ground-glass opacity (arrow). (B) Worst CXR first seen five days from time of obtaining the initial CXR (i.e. study period II), showing bilateral non-diffuse pattern of lower-lung-predominate mixed ground-glass and consolidative opacities (arrows). (C) Final CXR obtained 73 days from time of obtaining the initial CXR (i.e. study period VI), showing right lower lung ground-glass opacities and reticulations (arrow).
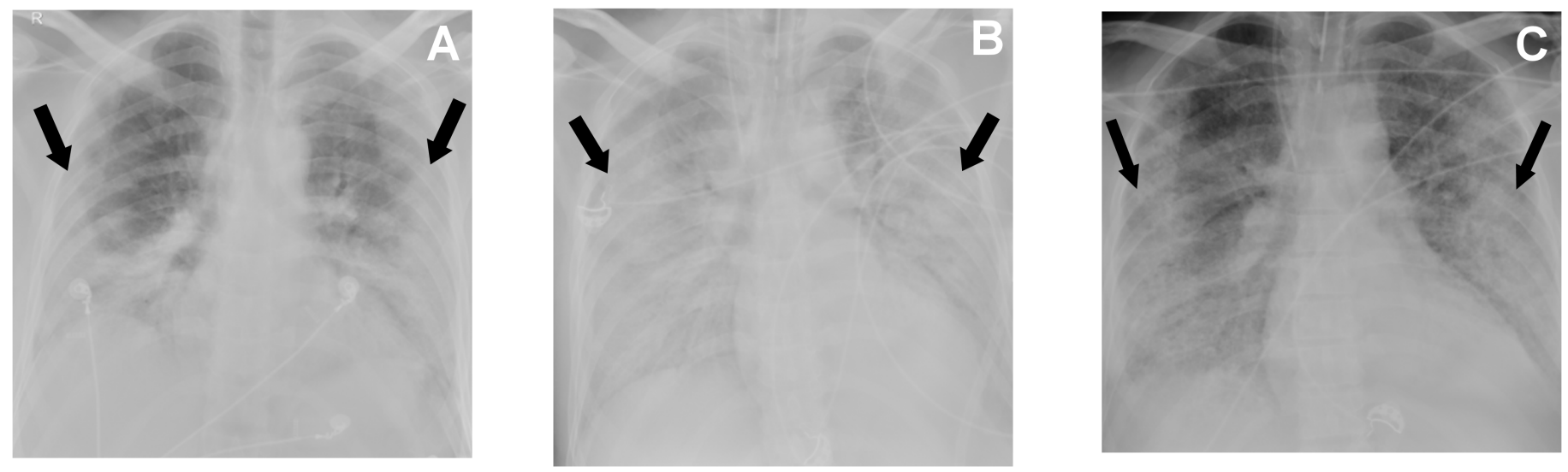

\section{Figure 2}

Figure 2. A 44-year-old male acquiring and dyeing from MERS-CoV infection after contact with camels. (A) Initial CXR obtained at eight days from symptoms onset, with bilateral non-diffuse pattern of peripheral and lower-lung-predominate mixed ground-glass and consolidative opacities (arrows). (B) Worst CXR first seen five days from time of obtaining the initial CXR (i.e. study period II), showing bilateral diffuse pattern of mixed ground-glass and consolidative opacities (arrows). (C) Final CXR obtained 15 days from time of obtaining the initial CXR (i.e. study period IV), showing bilateral non-diffuse pattern of peripheral and lower-lung-predominate mixed ground-glass and consolidative opacities (arrows). 

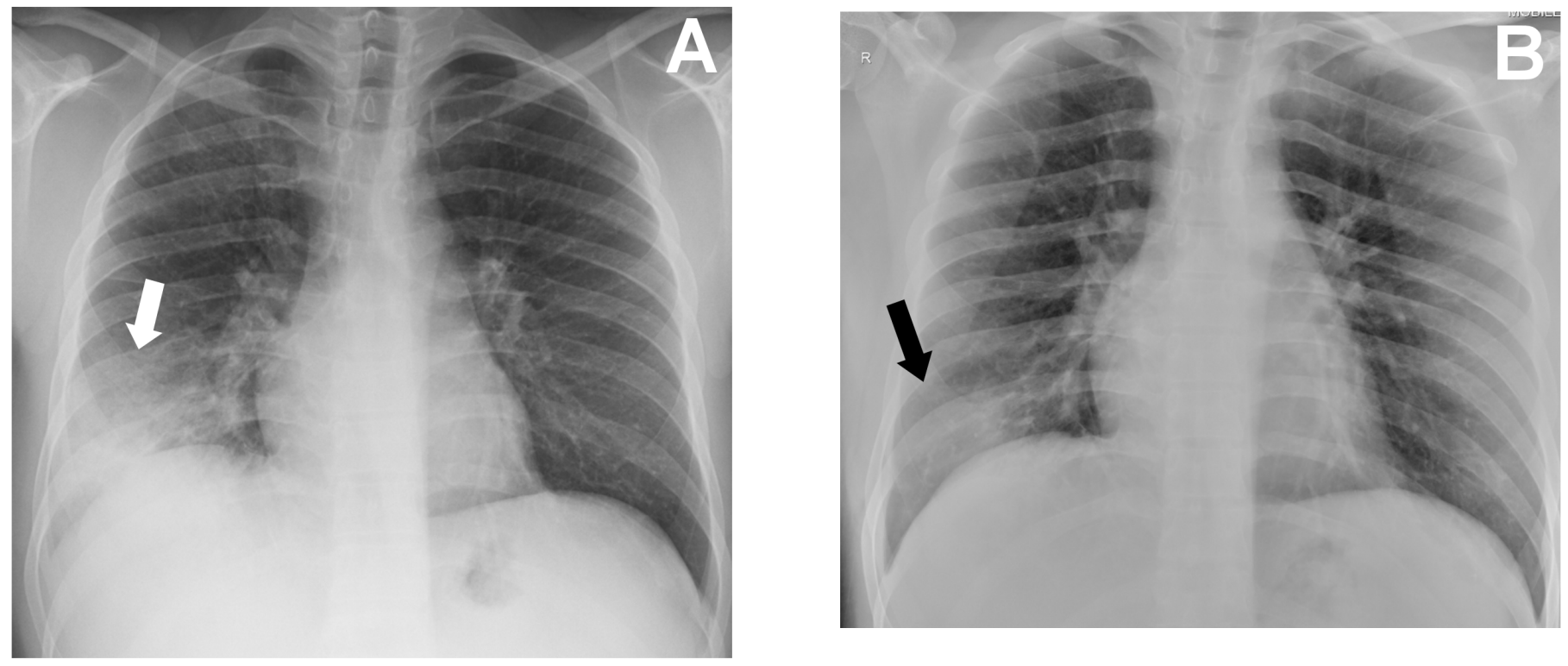

\section{Figure 3}

A 31-year-old male survivor of MERS-CoV infection, with an initial false-negative PCR result. (A) Initial CXR obtained at eight days from symptoms onset, showed the worst observed pattern in this patient, with focal right lower lung ground-glass opacity (arrow). (B) Final CXR obtained eight days from time of obtaining the initial CXR (i.e. study period III), showing residual focal right lower lung ground-glass opacity (arrow).
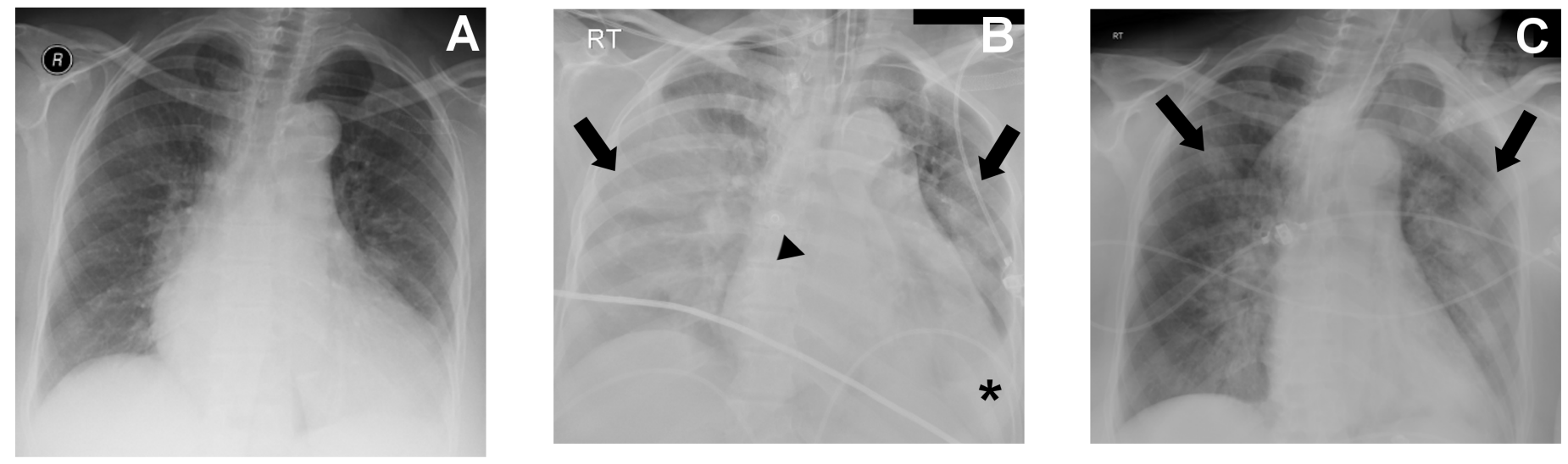

\section{Figure 4}

A 56-year-old female known for cardiac comorbidity, acquiring and dyeing from MERS-CoV infection after contact with another sick MERSCoV patient. (A) Initial CXR obtained at two days from symptoms onset, with normal lung and baseline cardiomegaly. (B) Worst CXR first seen three days from time of obtaining the initial CXR (i.e. study period II), showing bilateral diffuse pattern of heterogenous ground-glass opacities (thick arrows), focal left lower lobe consolidation (thin arrow) and left pleural effusion (arrowhead). (C) Final CXR obtained eight days from time of obtaining the initial CXR (i.e. study period III), showing bilateral diffuse pattern of residual heterogenous ground-glass opacities (arrows).

\section{Supplementary Files}

This is a list of supplementary files associated with this preprint. Click to download. 
- ajr.14.13021.pdf

Page 16/16 